\title{
Desarrollo de Arquitectura Empresarial usando un Framework con Enfoque Agil
}

\section{(Development of Enterprise Architecture using a Framework with Agile Approach) \\ Fanny Sandoval ${ }^{1}$, Víctor Galvez ${ }^{1}$, Oswaldo Moscoso ${ }^{1}$}

\begin{abstract}
Resumen:
El desarrollo de una arquitectura empresarial (AE) en grandes organizaciones es muy complejo. Por lo tanto, es importante que la implementación de la $A E$ cree valor en etapas tempranas del proceso para que los directivos apoyen esta iniciativa. Este documento presenta el diseño de un framework de AE que permite el desarrollo de arquitecturas con un enfoque ágil basado en TOGAF. Esta propuesta se la hace con el objetivo de agilizar el proceso de AE. Este framework plantea un nuevo diseño tomando en cuenta el marco regulatorio vigente, la línea destino de la organización y los principios propuestos por el enfoque ágil de AE. Los objetivos de cada etapa del método de desarrollo de arquitecturas ADM de TOGAF se cruzan con los requerimientos de la organización para extraer únicamente aquellos que se ajustan al giro del negocio. Se analiza los entregables propuestos por TOGAF con el fin de obtener, integrar y reducir la documentación en las fases de implementación y modelamiento, permitiendo mayor agilidad, menor impacto en los procesos, disminución en tiempos de desarrollo y costos.
\end{abstract}

Palabras clave: Arquitectura Empresarial; TOGAF; ADM; Enfoque Ágil; Estrategia Empresarial.

\begin{abstract}
:
The development of an enterprise architecture (EA) in large organizations is complex. Thus, is important that the implementation of EA creates value in early stages of the process. This document contains a proposal of an EA framework design with agile approach based in TOGAF. This proposal is done with the objective to streamline the EA process. This framework presents a new design taking into account the current regulations, the target line of the organization and the principles proposed by the agile approach of EA. The objectives of each phase of the architecture development method ADM of TOGAF are matched with the requirements of the organization to extract only those that are aligned to the business. The deliverables proposed by TOGAF are analyzed with the goal to obtain, integrate and reduce the documentation in the implementation and modeling phases. This reduction allows more flexibility, less impact in the processes, reduction in development time and costs.
\end{abstract}

Keywords: Enterprise Architecture; TOGAF; ADM; Agile Approach; Enterprise Strategy.

\section{Introducción}

Una organización es competitiva cuando sus componentes fundamentales están bien estructurados y cohesionados entre sí y con el ambiente (Koontz, Weihrich, \& Cannice, 2012). En la actualidad muchas organizaciones disponen de tecnologías de información que no están alineadas a los procesos, a los datos y a la planificación estratégica del negocio, otras disponen de sistemas legados de finanzas, compras, ventas, logística, recursos humanos, gestión de clientes. Esto

\footnotetext{
${ }^{1}$ Universidad Tecnológica Equinoccial Quito - Ecuador (\{fanny.sandoval, vhgalvez, omoscoso\}@ute.edu.ec)
} 
impide que estas entidades sean flexibles y ágiles. Consecuentemente estas instituciones no pueden adaptarse rápidamente a los cambios en el mercado y en el escenario global y político.

Es frecuente encontrar en cada unidad o departamento de una organización un centro de $\mathrm{TI}$ que atiende los requerimientos específicos de esa área, con sus propios estándares, marco regulatorio, entre otros, causando graves problemas en la gobernabilidad tecnológica, gastos onerosos y altos riesgos relacionados con los cambios.

En la actualidad la supervivencia de las organizaciones y su competitividad, depende de la globalización y de una nueva concepción económica que pretende: 1) Alcanzar un crecimiento inteligente, cuyo objetivo es desarrollar una economía fundamentada en el conocimiento y en la innovación, 2) Un crecimiento integrador que se basa en crear un alto nivel de empleo con cohesión social y territorial y 3) Un crecimiento sostenible, que se fundamenta en el uso eficaz de los recursos, que sea más competitiva y consciente de su responsabilidad con el medio ambiente (Regional, 2011).

Por lo tanto, las empresas deben adoptar nuevas formas organizativas, optimizar los recursos, establecer políticas y mejores prácticas con los clientes y facilitar la interoperabilidad de las tecnologías de código abierto. Para que las organizaciones puedan responder a estos desafíos ha surgido una nueva práctica llamada Arquitectura Empresarial $(\mathrm{AE})$, que es una metodología con una visión integral de la organización, permite alinear procesos, datos, aplicaciones e infraestructura tecnológica con los objetivos estratégicos de la organización.

Arquitectura Empresarial es una práctica estratégica continua dentro de la organización con objetivos bien definidos, que permite conectar todos los componentes de una empresa, apalancándose en la tecnología. Para llevarla a cabo existen diferentes marcos de trabajo o frameworks que ofrecen directrices y guías para el desarrollo e implementación de estrategias de AE en las organizaciones (Gonzáles \& Álzate, 2010). Cada framework tiene su propia estructura, estrategia y modelos de $\mathrm{AE}$.

Los frameworks empresariales más utilizados en el mundo son TOGAF y Zachman, pero difieren en el enfoque, composición y los términos de referencia. Zachman es un framework estructural y estático, es un esquema para organizar y categorizar. No tiene una metodología ni un modelo de referencia, por lo que su implementación es difícil.

Por otro lado TOGAF (The Open Group Architecture Framework) al ser un framework más liviano de implementar ayuda a reducir los costos, incrementar la calidad, identificar oportunidades y mejorar funcionalmente las aplicaciones. Además, reduce el riesgo, ya que identifica los objetivos de negocio y los involucrados en los diferentes dominios de arquitectura. Al mismo tiempo TOGAF es más práctico para crear una amplia variedad de 
arquitecturas empresariales sólidas, razón por la cual se toma como base para la realización de esta propuesta.

Consecuentemente el objetivo del presente trabajo es desarrollar un marco de trabajo de $\mathrm{AE}$ con enfoque ágil basado en TOGAF. TOGAF es uno de los frameworks más utilizados para planificar, diseñar, evaluar e implementar AE en una organización. Por otro lado, el enfoque ágil no determina un procedimiento detallado de cómo crear un tipo de modelo específico, sino que sugiere prácticas de modelado efectivo (Ambler P. S., 2002).

\section{TOGAF}

TOGAF es un framework abierto que comprende métodos y herramientas para la creación de Arquitecturas Empresariales (Ver Figura 1). Fue desarrollado en colaboración de un conjunto de empresas y agencias gubernamentales.

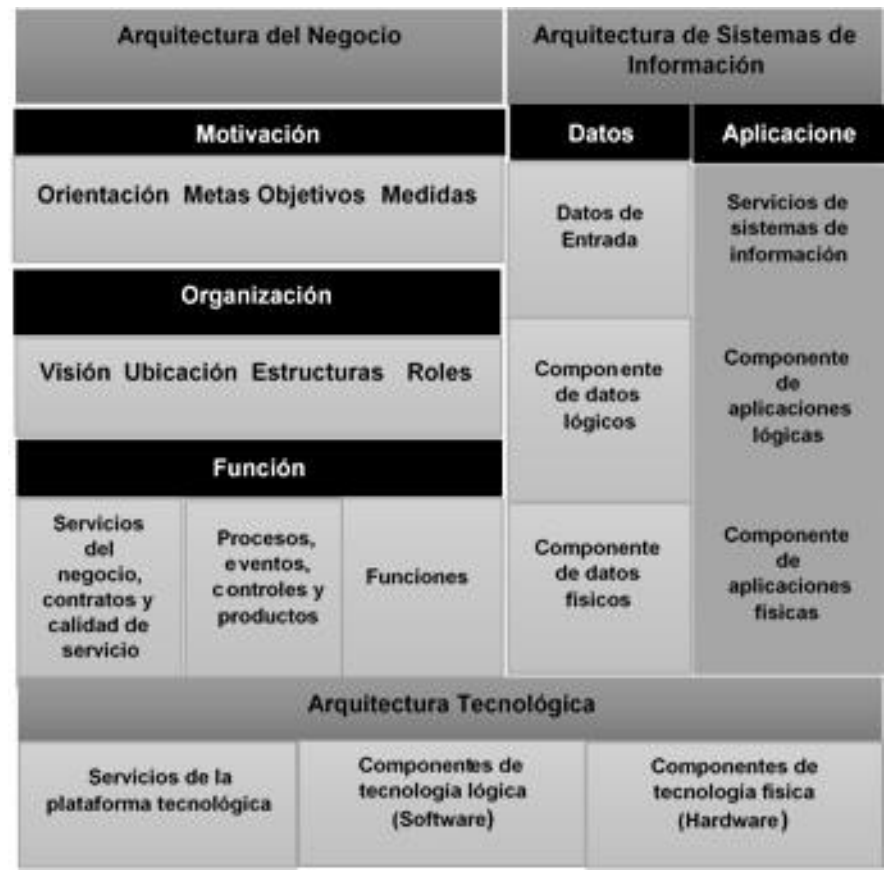

Figura 1. Capas de Arquitectura Empresarial

Se basa en un modelo iterativo respaldado por las mejores prácticas y un conjunto reutilizable de activos arquitectónicos existentes (Group, 2011), contiene tres dominios:

- Arquitectura de Negocios: Determina las estrategias de negocios, organización, gobernanza y procesos claves del negocio.

- Arquitectura de Sistemas de Información: comprende arquitectura de aplicaciones que detalla las aplicaciones existentes del negocio y sus iteraciones. Además, comprende la arquitectura de datos que determina cómo administrar los datos del negocio. 
- Arquitectura de Tecnología: Describe los componentes de hardware, software, comunicaciones y de redes que requiere el núcleo del negocio.

Maneja los siguientes componentes: Método de Desarrollo de Arquitectura (ADM), Continuum Empresarial y Repositorio de la Arquitectura. Tiene muchas ventajas entre las que se encuentran reducción de costos y reducción de riesgos; además:

- Permite identificar oportunidades.

- La empresa es más flexible y adaptable.

- Asegura que todos los involucrados hablen el mismo idioma.

El Método de Desarrollo de Arquitectura (ADM), es un conjunto de guías y técnicas para desarrollar AE. Es un método confiable que asegura el manejo adecuado de los requerimientos.

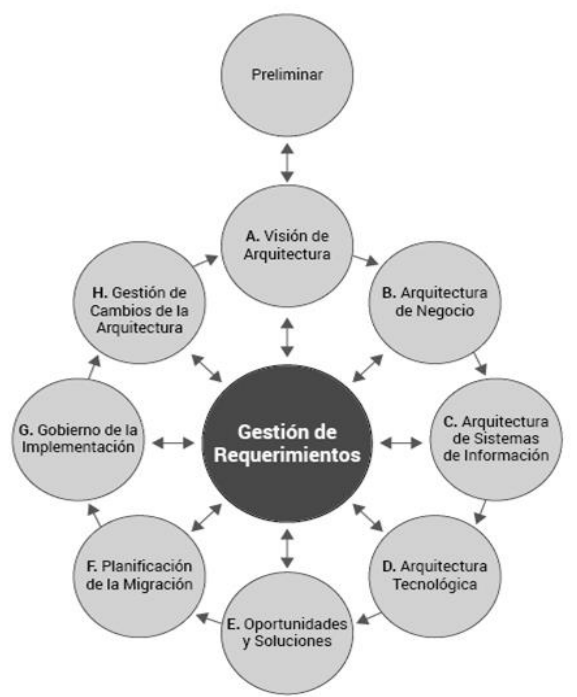

Figura 2. TOGAF V. 9.1.1 (Bricknal, Darrell, Nilsson, \& Pessi, 2011)

El ADM está constituido por varias fases como se puede apreciar en la Figura 2, las mismas que se desplazan a través de los dominios de la arquitectura, siendo éstas:

Fase Preliminar: Es una de las fases más críticas ya que se determina el ámbito del negocio que abarca la AE. Se define el alcance del proyecto y la estructura de gobernanza.

Fase A: Visión de la Arquitectura: esta es la primera fase de la arquitectura, en dónde se identifica las partes interesadas, sus expectativas y los requerimientos del negocio. La gerencia define las limitaciones y la visión de AE.

Fase B: Arquitectura de Negocio: en esta fase se determina la estructura de la organización, los procesos, los objetivos de negocio, sus funciones, servicios y sus roles. 
Fase C: Arquitectura de Sistemas de Información: determina los sistemas de información de la organización, las aplicaciones que los procesan, las relaciones entre ellos y con el ambiente, al igual que los procesos que gobiernan su diseño y evolución.

Fase D: Arquitectura Tecnológica: define el hardware, protocolos, sistemas de soporte para desarrollar los sistemas de información y tecnología de comunicaciones.

Fase E: Oportunidades y Soluciones: elabora el plan de implementación y se especifica cómo se va a realizar. Se decide si se debe: construir, comprar o reusar.

Fase F: Planeación de la Migración: se realiza un plan de migración detallado con un análisis costo/beneficio y un diagnóstico de los riesgos.

Fase G: Gobierno de la Implementación: se supervisa la implementación de la arquitectura para que cumpla con las especificaciones, normas y políticas.

Fase H: Gestión de Cambios de la Arquitectura: gestiona el cambio de la arquitectura de manera integrada e inteligente.

Gestión de Requerimientos: se identifica y gestiona los requerimientos al inicio y al final de cada fase del ADM

\section{Enfoque ágil}

Los marcos de trabajo existentes de Arquitectura Empresarial son muy amplios (Bricknal, Darrell, Nilsson, \& Pessi, 2011). Por lo tanto, no ofrecen ni la flexibilidad, ni la velocidad de entrega que las organizaciones requieren. Además, su desarrollo y puesta en marcha se fundamenta en fases y cada una de ellas tiene varios entregables. Esto implica un extenso tiempo de implementación que contradice a los principios de agilidad que exige el vertiginoso desarrollo de las TIC y los requerimientos del mercado.

Las organizaciones deben optimizar sus procesos para proporcionar productos y servicios de óptima calidad en el menor tiempo al mercado nacional e internacional (Blom, 2003). Por otra parte, están sujetas a cambios por la innovación digital, cloud computing, inteligencia del negocio, aplicaciones móviles, entre otros. Para poder gestionar los cambios de forma adecuada se necesita de un enfoque ágil (Ver Figura 3). Las características del enfoque ágil son las siguientes (Ambler S. , 2002):

- El trabajo se lo realiza en equipos motivados y la comunicación es cara a cara, analizando regularmente como ser más eficientes.

- Pone énfasis en la satisfacción de los clientes entregando los productos con valor en forma oportuna y continua.

- Mejorar la contribución de TI para alcanzar el ROI que las organizaciones requieren. 
- Aplicar los artefactos correctos para modelar en pequeños incrementos reutilizando los recursos existentes.

- Valorar a las personas e interacciones sobre los procesos y las herramientas.

- Mantener la AE simple. El modelado y la documentación tiene que ser únicamente lo necesario.

- Tener una visión completa de los pilares de la AE.

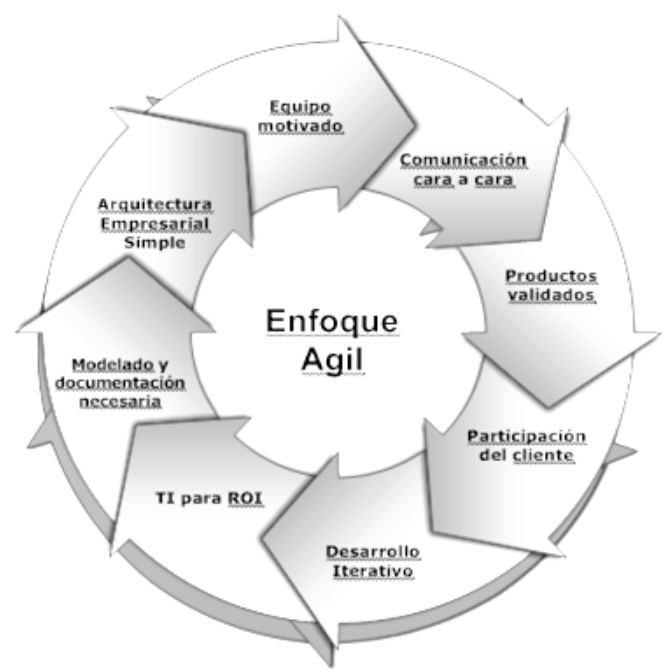

Figura 3. Proceso de Desarrollo Ágil orientado por el modelado

Principios ágiles resumidos:

Interacción: La interacción se manifiesta entre los diferentes actores de la Arquitectura Empresarial y de éstos con los clientes, los diseñadores trabajan en diferentes módulos y luego los integran con la retroalimentación de los clientes.

El cliente interactúa desde su sitio de trabajo y por medio de reuniones de trabajo permanentes se genera una retroalimentación, lo que le permite conocer lo que se realizó, qué problemas se presentaron y las actividades a realizarse.

Simplicidad: La arquitectura empresarial modelada y documentada tiene que ser justo lo que se necesita y a la vez ser susceptible a cambios continuos. Mantener estos artefactos lo más simples posible facilitará la comunicación con los stakeholders.

- El contenido es más importante, no importa el soporte físico o la técnica de representación.

- El cambio debe ser incremental.

- Producir sólo el modelo que resulte suficiente para el propósito.

- Maximizar la inversión del cliente.

- Modelos múltiples, múltiples paradigmas en convivencia, según se requiera. 
Retroalimentación: Trabajar iterativa e incrementalmente. Se propone la utilización de técnicas de modelado ágiles como "Aplicar el artefacto correcto", "Iterar a otro artefacto", "Crear varios modelos en paralelo" y "Modelar en pequeños incrementos" a la hora de modelar.

Respuesta al cambio: En un mundo globalizado nada permanece estático y las organizaciones pueden mantenerse competitivas gracias a la Arquitectura Empresarial Ágil, ya que ésta es intuitiva y ofrece agilidad, escalabilidad, y calidad requerida por los clientes.

El análisis del impacto de los cambios permitirá priorizar los proyectos que proporcionen mayor valor a la organización, además facilitará la implementación de nuevos modelos de negocios confiables y oportunos, lo que ocasionará una reducción de los costos.

En conclusión, se debe cumplir con todos los principios propuestos por el manifiesto ágil, los mismos que se pueden sintetizar en la Tabla 1:

Tabla 0. Principios del manifiesto ágil

\begin{tabular}{|c|c|}
\hline Interacción & $\begin{array}{l}\text { Las personas y su interacción por sobre las } \\
\text { herramientas } \\
\text { Y los procesos. }\end{array}$ \\
\hline Simplicidad & La funcionalidad sobre la documentación excesiva \\
\hline Retroalimentación & $\begin{array}{l}\text { La colaboración con el cliente por sobre la negociación } \\
\text { contractual }\end{array}$ \\
\hline Respuesta al cambio & $\begin{array}{l}\text { La respuesta al cambio por sobre el seguimiento de un } \\
\text { plan }\end{array}$ \\
\hline
\end{tabular}

\section{Diseño del framework}

El diseño de este framework se basó en una investigación cualitativa y exploratoria a través de un caso de estudio desarrollado los departamentos de investigación de dos instituciones de educación superior. Los pasos validados de diseño son los siguientes:

\section{A. Primer paso}

El diseño del framework comienza con la definición de los requerimientos de los stakeholders de la organización de acuerdo al giro del negocio, en base a tres premisas:

- Los fundamentos del enfoque ágil de $A E$ que se ajusten al modelo del negocio y a la visión de la organización, lo que permitirá que el nuevo framework sea simple, describa lo que sucede en la empresa y en su entorno. Además que el arquitecto empresarial que tenga una capacidad de respuesta rápida ante los cambios y se adapte con facilidad a cualquier nueva tendencia en el mercado.

- El marco legal de la organización y del ámbito de influencia del mismo.

- La línea destino de la organización la misma que se basa en la misión y visión de la organización. La base del diseño del nuevo framework es TOGAF, ya que ofrece una 
Metodología de Desarrollo de Arquitecturas o ADM, el cual proporciona un método probado y repetible para desarrollar Arquitecturas Empresariales.

\section{B. Segundo paso}

Para diseñar el framework se utiliza una matriz de cruce (Tabla 2). En está matriz los objetivos generales y específicos de cada fase del ciclo del método de desarrollo de arquitecturas ADM de TOGAF (Ver Figura 2) se cruzan con los requerimientos definidos en el primer paso, con el fin de:

- Eliminar los objetivos que no se cruzan con los requerimientos.

- Unificar los que tienen características comunes dentro de una misma fase.

Tabla 2. Cruce de requerimientos y objetivos específicos de las fases del ADM

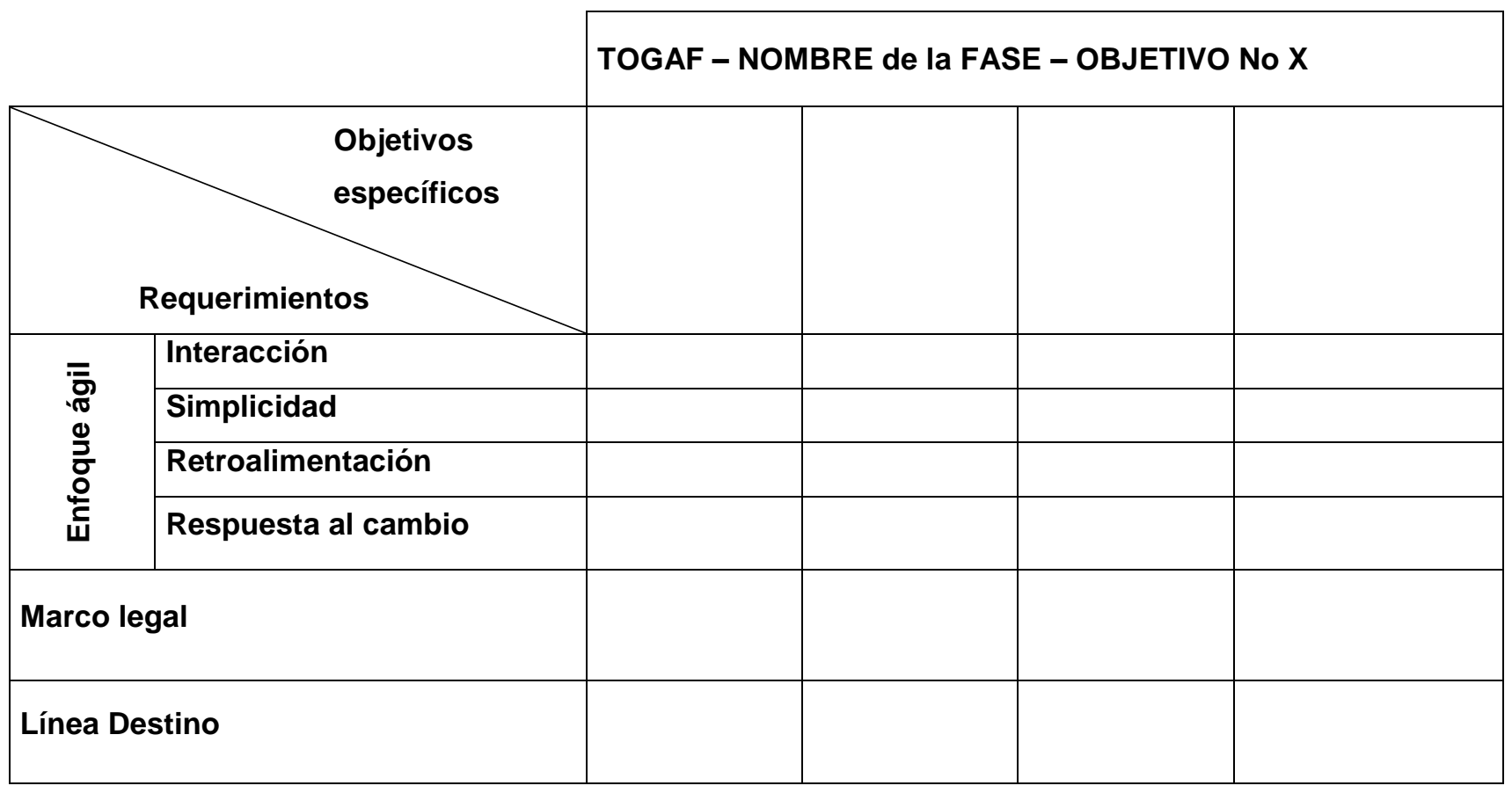

En la Tabla 3 se ejemplifica el cruce de los requerimientos con los objetivos específicos del objetivo "Determinar las Capacidades Arquitectónicas deseadas por la organización". Este objetivo corresponde a la Fase Preliminar del ADM.

Tabla 3. Cruce de los requerimientos con los objetivos específicos del primer objetivo de la fase Preliminar del ADM

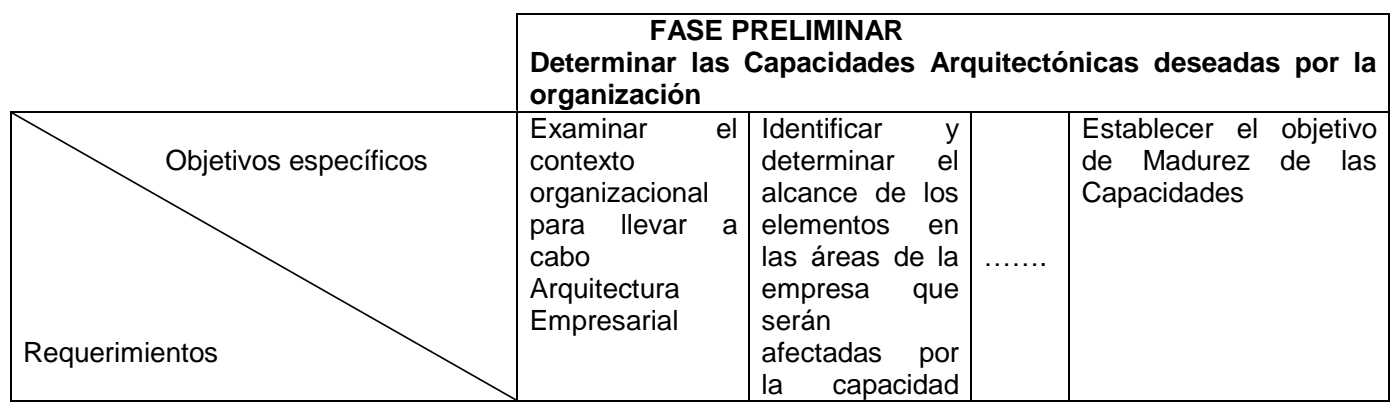




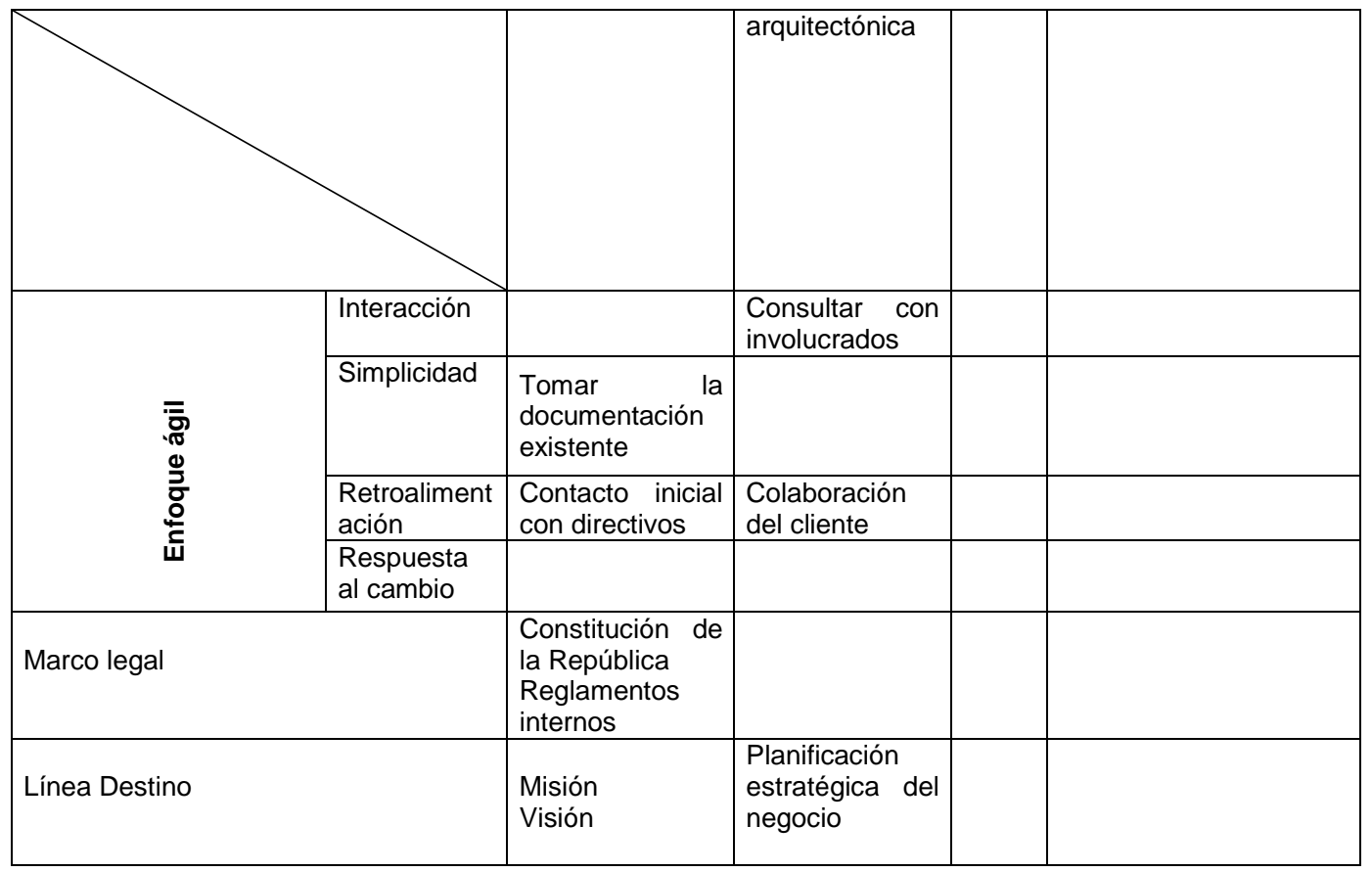

\section{Tercer paso}

En el tercer paso se analiza los entregables de cada fase propuestos por TOGAF. Un resumen de los entregables propuestos de acuerdo a cada fase del ADM se puede observar en la Tabla 4. La Tabla 5 por otro lado muestra un análisis de lo que implica la realización de los entregables. El detalle completo de los entregables se los puede observar en el libro de TOGAF.

Tabla 4. Entregables de cada fase de TOGAF

\begin{tabular}{|l|l|l|}
\cline { 2 - 3 } \multicolumn{1}{l|}{} & Entregable & Fases del ADM \\
\hline 1 & Marco de Referencia de Arquitectura Adaptado & Preliminar \\
\hline 2 & Modelo organizacional de Arquitectura Empresarial & Preliminar \\
\hline & $\cdot$ & \\
\hline 8 & $\cdot$ & A \\
\hline 9 & Visión de la Arquitectura & A \\
\hline 13 & Evaluación de Capacidades & A \\
\hline 14 & Gestión de Riesgos & A \\
\hline 15 & Documento de Definición de Arquitectura & A,B,C,D \\
\hline 30 & Solicitudes de Cambio & G,H \\
\hline 31 & Evaluación de Conformidad & G,H \\
\hline 32 & Evaluación del Impacto de los Requerimientos & H \\
\hline
\end{tabular}


Tabla 5. Análisis de Entregables

\begin{tabular}{|l|l|}
\hline DESCRIPCIÓN & ANÁLISIS \\
\hline 1. Marco de Referencia de Arquitectura Adaptado \\
$\begin{array}{l}\text { Se encarga de adaptar el modelo TOGAF para la } \\
\text { integración a la empresa. }\end{array}$ & $\begin{array}{l}\text { El presente proyecto, reformula el framework para ser } \\
\text { adaptado. }\end{array}$ \\
\hline 2. Modelo organizacional de Arquitectura Empresarial \\
$\begin{array}{l}\text { Define la organización, roles y las responsabilidades } \\
\text { apropiadas dentro de la empresa. }\end{array}$ & Se conserva. \\
\hline $\begin{array}{l}\text { - } \\
\text { - }\end{array}$ & \\
\hline 32. Evaluación del Impacto de los Requerimientos \\
\hline $\begin{array}{l}\text { Evalúa los requerimientos y especificación actual de } \\
\text { arquitectura para identificar los cambios que se deben } \\
\text { hacer y las consecuencias derivadas de su introducción }\end{array}$ & Es necesaria, se conserva. \\
\hline
\end{tabular}

Como se pude observar, algunos entregables pueden ser omitidos, ya que salen del alcance del proyecto, o porque pueden ser fusionados con otros entregables. En el caso de estudio realizado de 32 entregables sugeridos por TOGAF se redujo a 19 entregables de acuerdo al enfoque ágil propuesto en este trabajo. Un ejemplo de esta reducción se muestra en la Tabla 6.

Tabla 6. Resumen de los entregables luego de la reducción

\begin{tabular}{|c|c|c|c|}
\hline No & Entregable & Incluye & Fase que se utiliza \\
\hline 1 & Estudio preliminar & $\begin{array}{l}\text { 1.1 Modelo organizacional de AE } \\
\text { 1.2 Principios de Arquitectura } \\
\text { 1.3 Repositorio de Arquitectura } \\
\text { 1.4 Herramientas de Arquitectura }\end{array}$ & Preliminar \\
\hline 2 & $\begin{array}{l}\text { Petición de Trabajo de } \\
\text { Arquitectura }\end{array}$ & & Preliminar \\
\hline 3 & $\begin{array}{l}\text { Declaración de Trabajo de } \\
\text { Arquitectura }\end{array}$ & $\begin{array}{l}\text { 3.1 Declaración de Trabajo de } \\
\text { Arquitectura y Visión de la Arquitectura }\end{array}$ & $A$ \\
\hline$\dot{.}$ & & & \\
\hline 18 & Evaluación de Conformidad & & $\mathrm{G}, \mathrm{H}$ \\
\hline 19 & $\begin{array}{l}\text { Evaluación del Impacto de los } \\
\text { Requerimientos }\end{array}$ & & $\begin{array}{l}\text { Gestión } \\
\text { Requerimientos }\end{array}$ \\
\hline
\end{tabular}




\section{Cuarto paso}

Con el fin de tener mayor simplicidad y agilidad en el desarrollo de la $A E$, los entregables resultantes del análisis del tercer paso se cruzan con los objetivos de las fases del ADM que quedaron en el segundo paso (luego de eliminar aquellos objetivos que no coincidían con los requerimientos). De esa forma la $A E$ se enfoca únicamente en realizar las tareas que vayan de acuerdo a los entregables que están dentro del alcance del proyecto y de los objetivos de la organización.

\section{E. Quinto paso}

En el quinto paso se detalla los objetivos y entregables que se obtuvieron de la simplificación realizada en los pasos anteriores. Los pasos del diseño fueron validados como caso de estudio en una institución de educación superior. Una muestra del resultado de la aplicación del framework y la reducción e integración realizada en la fase preliminar se muestra a continuación:

\section{FASE PRELIMINAR}

\section{Objetivos}

\section{- Determinar las capacidades arquitectónicas deseadas por la organización}

a) Examinar el contexto organizacional para llevar a cabo $\mathrm{AE}$

b) Identificar y determinar el alcance de los elementos en las organizaciones que serán afectadas por la capacidad arquitectónica

c) Identificar los marcos de referencia establecidos, los métodos y los procesos que se entrecruzan con la capacidad arquitectónica

\section{- Establecer las Capacidades Arquitectónicas}

a) Definir y establecer el modelo organizacional de AE.

b) Definir y establecer el proceso detallado, los recursos y las herramientas para el gobierno de la arquitectura

c) Definir los principios de arquitectura

\section{Entregables: 1, 2}

\section{Conclusiones y discusión}

Esta metodología simplifica la definición y el desarrollo de arquitecturas, además permite a la organización realizar la transferencia de la tecnología, gestionar adecuadamente los proyectos y contar con indicadores de desempeño y reconocimiento. 
Este framework con enfoque ágil, es una metodología de mejora continua a mediano plazo. La organización que implemente este framework, estará en capacidad de aprovechar las oportunidades que presenta el mercado y el desarrollo tecnológico. Un framework de AE que usa el enfoque ágil permite:

- Usar en forma efectiva los recursos, lo que se traduce en un ahorro importante para la organización.

- Responder eficientemente a los retos y oportunidades que presenta el mercado, los cambios tecnológicos y cualquier otra circunstancia planificada o inesperada.

- Estandarizar y mejorar procesos del negocio y de TI.

- La AE es proactiva ya que identifica oportunidades de integración y de reutilización de aplicaciones y recursos en toda la organización

- Contar con indicadores para la toma de decisiones.

- Mejorar la satisfacción del cliente.

- Manejar cambios organizacionales, restructuraciones, fusiones y adquisiciones.

- El enfoque ágil en $A E$ es un proceso en el que se aplican un conjunto de buenas prácticas para trabajar colaborativamente, en equipo, y obtener el mejor resultado de un proyecto.

A pesar de que las perspectivas de mejora en tiempos y calidad de implementación de arquitecturas empresariales usando el framework propuesto son altas, sería importante aplicarlo en diferentes tipos de instituciones para poder valorar su aplicabilidad en un contexto global.

\section{Bibliografía}

Ambler, P. S. (2002). Modelado Ágil ( AM ) Valores Principios Prácticas.

Ambler, S. (2002). Agile Modeling: Effective Practices for eXtreme Programming and the Unified Process.

Blom, A. C. (2003). Enterprise Modernization (Vol. 7), No. 2, 2003.

Bricknal, R., Darrell, G., Nilsson, H., \& Pessi, K. (2011). Enterprise Architecture: Critical Factors affecting modelling and management.

Gonzáles, E., \& Álzate, J. (2010). Frameworks de Arquitectura Empresarial. Retrieved Aug 20, 2016, from https://arquitecturaempresarialcali.wordpress.com/2010/11/16/frameworks-dearquitectura-empresarial/

Group, T. O. (2011). TOGAF ${ }^{\circledR}$ Version 9.1. Retrieved from http://goo.gl/djuv15. 
Koontz, H., Weihrich, H., \& Cannice, M. (2012). Administración una Perspectiva Global y Empresarial. Mc Graw Hill 14ava ed..

Regional, U. E. (2011). Alcanzar un crecimiento inteligente, sostenible e integrador (Vol. 36). Panor. Inforegio. No. Vol 36, 2011 